\title{
Lesão do manguito rotador em cadeirantes com lesão medular: 0 tempo de lesão e o nível medular interferem? Uma avaliação retrospectiva
}

\section{Rotator Cuff Lesion in Wheelchair Users With Spinal Cord Injury: Does Time of Injury and Medullary Level Interfere?}

\section{A Retrospective Evaluation}

\author{
Cládis Sanches Lopes Filho ${ }^{10}$ Mauro Coura Perez ${ }^{10}$ Pedro Cordeiro Moraes ${ }^{1(0)}$ \\ Gabriel Costa Serrão de Araújo ${ }^{10}$ \\ ${ }^{1}$ Cirurgia Ortopédica, Rede SARAH de Hospitais de Reabilitação, \\ Brasília, DF, Brasil \\ Rev Bras Ortop 2022;57(4):584-589. \\ Endereço para correspondência Cládis Sanches Lopes Filho, MD, \\ Rede SARAH de Hospitais de Reabilitação, SMHS QD 501 Bloco A, \\ Brasília, DF, Brasil (e-mail: cladislopes@hotmail.com; \\ cladis@sarah.br).
}

\begin{abstract}
Resumo
Objetivo Descrever as características de ressonância magnética (RM) dos ombros de pacientes com lesão medular (LM) e correlacionar esses achados com idade, duração da LM e nível neurológico.

Método A amostra do presente estudo incluiu pacientes maiores de 18 anos com LM torácica, que eram cadeirantes ativos e haviam sido submetidos a uma ressonância magnética do ombro de janeiro de 2004 a dezembro de 2015.

Resultados Foram estudados 41 ombros (37 pacientes), incluindo 27 de pacientes do sexo masculino $(65,9 \%)$ e 14 de pacientes do sexo feminino (34,1\%). Na época da ressonância magnética, a média de idade era de 41,9 anos e a duração média da LM era de 9,4 anos. A análise da relação entre a duração do trauma e a gravidade da lesão do

Palavras-chave

- imagem de ressonância magnética

- ombro

- traumatismos da medula espinal

- cadeira de rodas manguito rotador (LMR), bem como entre a idade e a gravidade da lesão do ombro mostrou diferença estatisticamente significativa $(p<0,001)$, com associação positiva em ambos os casos. Não foi observada diferença estatisticamente significativa $(p=0,095)$ entre o nível neurológico da LM e da LMR.

Conclusão Neste estudo, pode-se notar um aumento progressivo da gravidade das lesões do ombro com o avanço da idade e uma maior duração da LM. No entanto, o nível da LM não parece interferir com a LMR.

Nível de Evidência Nível IV, série de casos.
\end{abstract}

Trabalho desenvolvido na Rede SARAH de Hospitais de Reabilitação, Brasília, DF, Brasil.

recebido

05 de Agosto de 2020

aceito

02 de Outubro de 2020

Publicado on-line

Abril 19, 2021
DOI https://doi.org/ $10.1055 / \mathrm{s}-0041-1724081$ ISSN 0102-3616.
(C) 2021. Sociedade Brasileira de Ortopedia e Traumatologia. All rights reserved.

This is an open access article published by Thieme under the terms of the Creative Commons Attribution-NonDerivative-NonCommercial-License, permitting copying and reproduction so long as the original work is given appropriate credit. Contents may not be used for commercial purposes, or adapted, remixed, transformed or built upon. (https://creativecommons.org/ licenses/by-nc-nd/4.0/)

Thieme Revinter Publicações Ltda., Rua do Matoso 170, Rio de Janeiro, RJ, CEP 20270-135, Brazil 


\begin{abstract}
Keywords

- magnetic resonance imaging

- shoulder

- spinal cord injuries

- wheelchairs

Objective To describe the characteristics of magnetic resonance imaging (MRI) of the shoulders of patients with spinal cord injury $(\mathrm{SCl})$ and to correlate these findings with age duration of $\mathrm{SCl}$ and neurological level.

Method The study sample included patients with thoracic SCl over 18 years of age who were active wheelchair users and had undergone an MRI of the shoulder from January 2004 to December 2015.

Results We studied 41 shoulders (37 patients) including 27 of male patients (65.9\%) and 14 of female patients (34.1\%). At the time of MRI the mean age was 41.9 years and the mean duration of $\mathrm{SCl}$ was 9.4 years. The analysis of the relationship between the duration of trauma and severity of the rotator cuff lesion ( $R C L$ ) as well as between age and severity of the shoulder injury showed a statistically significant difference $(p<0.001)$ with a positive association in both cases. No statistically significant difference ( $p=0.095)$ was observed between the neurological level of the SCI and RCL. Conclusion In this study a progressive increase in the severity of the shoulder lesions can be noted with advancing age and a longer duration of SCl. However the level of the $\mathrm{SCl}$ does not seem to interfere with the RCL.

Level of Evidence Level IV case series.
\end{abstract}

\section{Introdução}

No dia a dia, pessoas com lesão medular (LM) necessitam do uso de membros superiores para transferências e propulsão ao usar suas cadeiras de rodas. Consequentemente, espera-se que, com o passar do tempo, dores nas articulações e lesões no ombro ocorram. ${ }^{1-4}$ As cinemáticas escapulotorácicas e glenoumerais das atividades, incluindo transferências e levantamento de peso corporal em pessoas com LM, já foram investigadas anteriormente. Estes estudos têm demonstrado que os padrões posicionais estão associados à diminuição do espaço subacromial e ao aparecimento de lesões anterossuperiores do manguito rotador. ${ }^{5,6}$

Este estudo tem como objetivo descrever as características do ombro dos cadeirantes com LM torácica que foram tratados em nossa instituição, analisando os achados de ressonância magnética (RM) do ombro desses pacientes.

\section{Materiais e métodos}

Este estudo é uma análise observacional, transversal (série de casos) de pacientes em cadeira de rodas manual com LM torácica, acima de 18 anos, submetidos à RM do ombro, devido a queixas de dor nas articulações, de janeiro de 2004 a dezembro de 2015. Encontramos 37 pacientes com um total de 41 ombros. Foi realizada uma análise de prontuário eletrônico para determinar o sexo, a idade no momento da $\mathrm{RM}$, a causa da LM, o nível neurológico (de acordo com os critérios da American Spinal Injury Association [ASIA]), ${ }^{7}$ o tempo decorrido da LM até a data do exame de ressonância magnética e informações sobre possíveis cirurgias no ombro. Todas as RMs foram realizadas em equipamentos de $1.5 \mathrm{~T}$. Todas as imagens foram simultaneamente revisadas por dois autores durante a análise dos prontuários., e identificaram presença ou ausência de artrite da articulação glenohumeral
(GH) e da articulação acromioclavicular (AC), tipo de acrômio classificado de acordo com Bigliani et al., ${ }^{8}$ e presença ou ausência de bursite. Os tendões dos músculos supraespinhal, infraespinhal e subescapular foram analisados para a presença de tendinite, lesões parciais ou completas. $O$ estado de degeneração gordurosa da massa muscular envolvida foi avaliado de acordo com a classificação Goutalier. ${ }^{9}$

Os dados coletados foram inseridos nas planilhas do Microsoft Excel (Microsoft Corp. Redmond, WA, EUA), também utilizadas na elaboração de gráficos. As análises estatísticas foram concluídas usando a versão 21 do IBM SPSS para Windows (IBM Corp., Armonk, NY, EUA). Para caracterização da amostra, foi feita uma análise descritiva dos resultados das variáveis. A variabilidade da distribuição de uma variável quantitativa foi considerada baixa se o coeficiente de variação (CV) $<0,20$; moderado se $0,20 \leq \mathrm{CV}<0,40$, e alto se o $\mathrm{CV}$ $\geq 0,40$. As amostras foram testadas para normalidade com $o$ teste de Shapiro-Wilk, que não revelou normalidade nas distribuições de idade e tempo de trauma. Dada a ausência de normalidade e nível de natureza ordinais de gravidade e nível neurológico da lesão, toda a abordagem inferencial foi não paramétrica. A associação entre duas variáveis qualitativas foi feita utilizando-se o teste do qui-quadrado ou, quando inconclusivo, o teste exato de Fisher foi utilizado. A medida utilizada para estimar o risco foi a razão de chances (RC). Na análise da associação entre uma variável quantitativa e uma qualitativa, a comparação de dois grupos independentes foi realizada pelo teste de Mann-Whitney e mais de dois grupos independentes foram comparados utilizando-se o teste de Kruskal-Wallis. A associação entre duas variáveis quantitativas foi investigada pela análise de correlação, verificando o gráfico de dispersão e calculando o coeficiente de correlação da ordem Spearman. A significância dos coeficientes de correlação foi avaliada pelo teste de coeficiente de correlação. A correlação entre duas variáveis foi considerada forte se o coeficiente de 
correlação apresentasse valor absoluto superior a 0,7, e moderado se seu valor absoluto estivesse na faixa de 0,3 a 0,7 . Todas as discussões sobre testes de significância foram realizadas considerando um nível máximo de significância de 5\% (0,050).

\section{Resultados}

De 41 ombros, $14(34,1 \%)$ eram de pacientes do sexo feminino e $27(65,9 \%)$ de pacientes do sexo masculino; no entanto, a diferença entre as proporções de homens e mulheres não foi significativa ( $p=0,064$, teste binomial); 25 casos (61,0\%) estavam do lado direito e 16 (39,0\%) do lado esquerdo, e a diferença entre essas proporções também não foi significativa $(p=0,211$, teste binomial). A idade dos participantes variou de 22 a 65 anos, com média de 41,9 anos (desvio padrão $[D P]=10,7 ; C V=0,26$ ), e o tempo de trauma medular até a RM realizada variou de 1 a 29 anos, com média de 9,4 anos ( $\mathrm{DP}=9,1 ; \mathrm{CV}=0,97$ ). Pelos valores dos CVs, observa-se que a amostra era homogênea em relação à idade, mas heterogênea considerando o tempo do trauma. As principais causas das lesões foram os acidentes de trânsito (29 casos; 70,7\%) e as quedas de altura (5 casos; $12,2 \%$ ). O nível neurológico da lesão foi classificado de acordo com a classificação da ASIA. Em resumo, 17 casos $(41,5 \%)$ foram de lesões torácicas elevadas (T2-T6) e 24 casos $(58,5 \%)$ foram de lesões torácicas baixas (T7-T12), e não houve diferença estatisticamente significativa entre essas duas proporções ( $p=0,533$, teste binômio).

Cada caso de lesão no ombro foi categorizado por nós em 14 estágios de gravidade, variando de ausência de lesão nos três músculos (menos grave) a dois rompimentos completos e um parcial (mais grave), como mostrado na - Tabela 1. Na análise

Tabela 1 Distribuição das frequências do nível de gravidade da lesão

\begin{tabular}{|l|l|l|l|}
\hline $\begin{array}{l}\text { Nível de } \\
\text { gravidade } \\
\text { da lesão }\end{array}$ & $\begin{array}{l}\text { Tipo de } \\
\text { lesão }\end{array}$ & Frequência & Porcentagem \\
\hline 0 & $0 \mathrm{~T}+0 \mathrm{RP}+0 \mathrm{RC}$ & 3 & 7,3 \\
\hline 1 & $1 \mathrm{~T}$ & 6 & 14,6 \\
\hline 2 & $2 \mathrm{~T}$ & 8 & 19,5 \\
\hline 3 & $3 \mathrm{~T}$ & 1 & 2,4 \\
\hline 4 & $1 \mathrm{PT}$ & 1 & 2,4 \\
\hline 5 & $1 \mathrm{RP}+1 \mathrm{~T}$ & 5 & 12,2 \\
\hline 6 & $1 \mathrm{RP}+2 \mathrm{~T}$ & 1 & 2,4 \\
\hline 7 & $2 \mathrm{RP}$ & 1 & 2,4 \\
\hline 8 & $2 \mathrm{RP}+1 \mathrm{~T}$ & 5 & 12,2 \\
\hline 9 & $3 \mathrm{RP}$ & 1 & 2,4 \\
\hline 10 & $1 \mathrm{RC}+1 \mathrm{~T}$ & 3 & 7,3 \\
\hline 11 & $1 \mathrm{RC}+1 \mathrm{RP}+1 \mathrm{~T}$ & 3 & 7,3 \\
\hline 12 & $1 \mathrm{RC}+2 \mathrm{RP}$ & 1 & 2,4 \\
\hline 13 & $2 \mathrm{RC}+1 \mathrm{~T}$ & 1 & 2,4 \\
\hline 14 & $2 \mathrm{RC}+1 \mathrm{RP}$ & 1 & 2,4 \\
\hline
\end{tabular}

Abreviaturas: RC, ruptura completa; RP, ruptura parcial; T, tendinopatia. descritiva da RM, a artrite GH foi encontrada em 9 ombros (22\%). Considerando a articulação AC, 28 ombros (68,3\%) foram caracterizados com artrite. Quanto à bursite subacromial, 33 ombros (80,5\%) tiveram algum processo inflamatório. A forma do acrômio foi tipo I em 16 (39,0\%), tipo II em 23 (56,1\%) e tipo III em 2 ombros (4,9\%). Na avaliação da lesão labral, apenas 10 ombros $(24,4 \%)$ apresentaram lesões em alguns segmentos labrais identificados. Ao avaliar os tendões que compõem o manguito rotador, excluindo o tendão menor, verificou-se que o tendão subescapular não foi afetado em 16 ombros (39,0\%), apresentou tendinopatia em 14 (34,2\%) e ruptura parcial em 11 ombros (26,8\%). 0 tendão supraespinhal não foi afetado em 6 ombros (14,6\%), apresentou tendinopatia em 16 (39,0\%), ruptura parcial em $10(24,4 \%)$ e ruptura completa em 9 ombros (22,0\%). Nos casos de ruptura completa do supraespinhal, o tamanho médio da ruptura foi de 21,3 $\mathrm{mm}(\mathrm{DP}=10,1 \mathrm{~mm}$, $\mathrm{CV}=0,47$, alta variabilidade). Esses casos, de acordo com a classificação de Goutalier, 1 (11,1\%), foram classificados como grau 0, 1 (11,1\%) como grau I, 4 (44,4\%) como Grau II, 1 (11,1\%) como grau III e $2(22,2 \%)$ como grau IV. Quanto ao infraespinhal, 14 ombros (34,2\%) apresentaram tendinopatia, $7(17,1 \%)$ apresentaram lesões parciais e apenas 2 ombros (4,9\%) apresentaram rasgo completo, um com 15 e outro com $23 \mathrm{~mm}$ de tamanho (média de 19,0 mm), e ambos foram classificados como grau I segundo a classificação de Goutalier.

A análise de correlação foi realizada entre as variáveis idade (no momento da ressonância magnética), tempo de lesão, nível neurológico de LM e nível de gravidade da lesão. Os lotes de dispersão são mostrados na - Fig. 1. Verificou-se que a gravidade da lesão do ombro está correlacionada com a idade dos participantes e com o tempo de LM, com correlações moderadas medidas pelo coeficiente de correlação da ordem de Spearman ( $r=0,544$ e $r=0,452$, respectivamente). Não houve correlação entre nível neurológico de LM e gravidade da LMR ( $p=0,095)$.

Observando os valores de $p$ encontrados na - Tabela 2, é possível concluir que a condição do tendão supraespinhal estava associada à idade $(p=0,006)$ e ao tempo de trauma $(p=0,038)$. Observa-se também que a idade média dos pacientes aumenta à medida que a lesão supraespinhal aumenta, a ponto de os pacientes com lesão completa serem significativamente mais velhos (média de 52,4 anos). A condição do infraespinhal não esteve associada à idade dos participantes $(p=0,062$, teste de Kruskal-Wallis), mas foi associada ao tempo de trauma ( $p=0,029$, teste de Kruskal-Wallis). Observa-se que os casos sem lesões no tendão infraespinhal foram de pacientes com tempo de lesão significativamente menor (média de 5,4 anos), e o tempo médio de lesão espinhal aumenta à medida que a complicação do infraespinhal aumenta, a ponto de os pacientes com lesão completa terem um tempo de lesão significativamente maior (média de 26,0 anos). A condição do tendão subescapular não foi associada com a idade dos participantes ( $p=0,050$, teste de Kruskal-Wallis) nem com o tempo de trauma ( $p=0,240$, teste de Kruskal-Wallis). Na avaliação geral do manguito rotador, considerando os 3 músculos, 23 ombros (56,1\%) apresentaram alguma lesão em pelo menos 1 dos 3 músculos avaliados e 18 ombros (43,9\%) não apresentaram lesões (-Tabela 2). Há uma diferença estatisticamente 

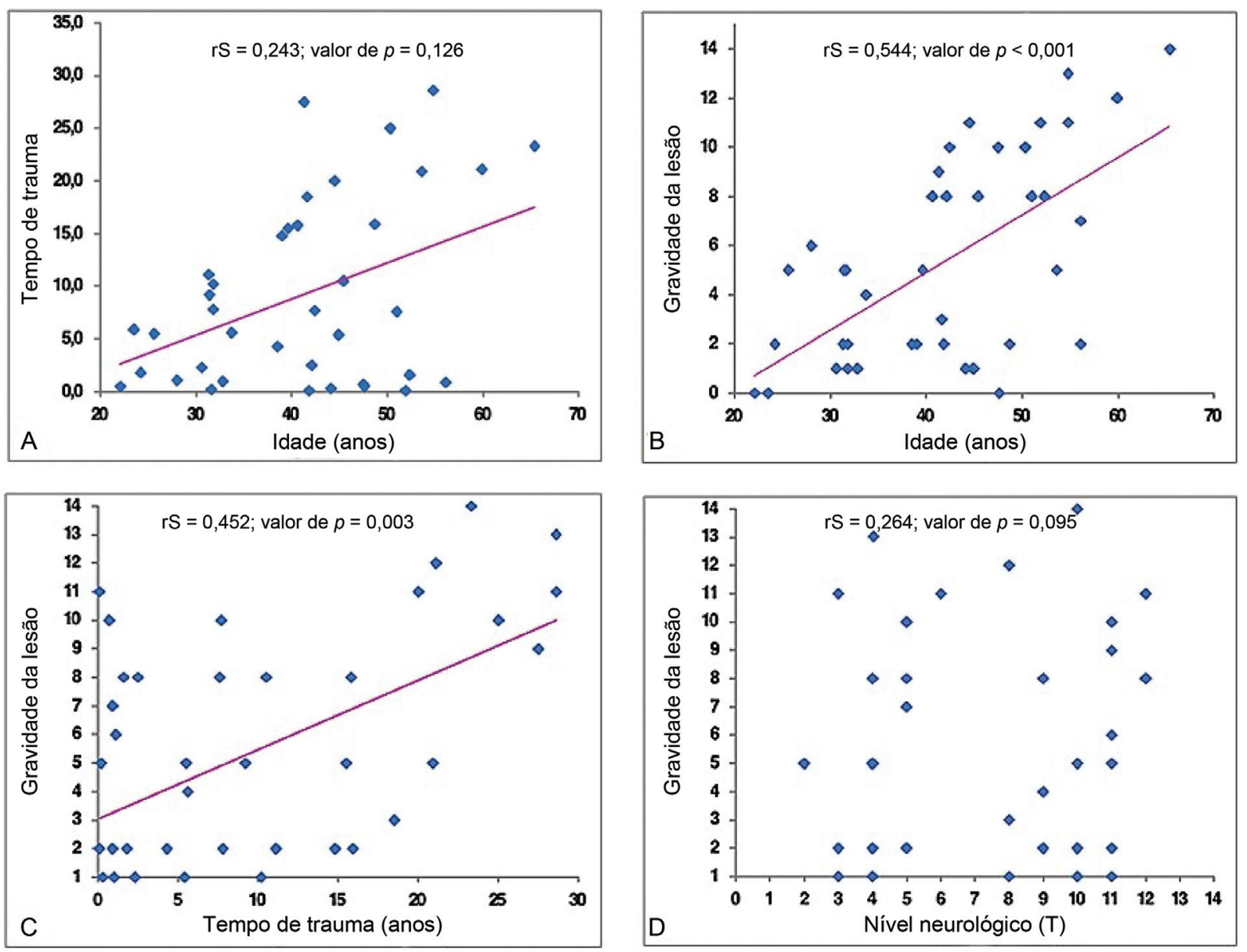

Fig. 1 Parcelas de dispersão • (A) Tempo de trauma (anos) x idade (anos). Há uma ligeira tendência positiva, mas com pontos distantes dessa linha de tendência. De fato, a correlação entre as duas variáveis é fraca $(\mathrm{rS}=0,243,<0,70)$ e não significativamente diferente de zero $(p=0,126)$

- (B) Gravidade da lesão x idade (anos). Há uma ligeira tendência positiva, mas com pontos distantes dessa linha de tendência. De fato, a correlação entre as duas variáveis é significativa $(p<0,001)$, indicando que à medida que a idade aumenta a gravidade da lesão tende a aumentar, mas a correlação não é forte $(r S=0,544,<0,70)$. (C) Gravidade da lesão versus tempo de trauma (anos). É possível verificar uma ligeira tendência positiva, mas com pontos muito distantes dessa linha de tendência. De fato, a correlação entre as duas variáveis é significativa $(p=0,003)$, indicando que o tempo de trauma aumenta, a gravidade da lesão tende a aumentar, mas a correlação não é forte $(r S=0,452,<0,70)$ - (D) Gravidade da lesão x nível neurológico (T). Não há linha de tendência, com correlação fraca entre as duas variáveis $(r S=0,264,<0,70)$ e não significativamente diferente de zero (valor de $p=0,095$ ).

significativa entre a idade dos pacientes que não apresentaram lesões nos 3 músculos e os pacientes que apresentaram lesões em pelo menos 1 músculo ( $p=0,023$, teste de Mann-Whitney). Não houve diferença estatisticamente significativa entre 0 tempo de LM em casos sem lesão nos 3 músculos e nos casos com lesão em pelo menos 1 músculo ( $p=0,057$, teste de MannWhitney). A artrose GH está significativamente associada ao tempo de trauma ( $p=0,020$, teste de Mann-Whitney). 0 tempo médio de trauma do grupo que não tinha artrose $\mathrm{GH}$ foi de 7,3 anos, com desvio-padrão de 7,2 anos e mediana de 5,4 anos. No grupo que tinha artrose $\mathrm{GH}$, o tempo de trauma foi significativamente maior; média $=16,9$ anos, desvio-padrão $=11,5$ anos, mediana $=20$ anos. A artrose $\mathrm{GH}$ foi significativamente associada à gravidade da lesão no manguito ( $p=0,003$, teste de Mann-Whitney). No grupo sem artrose $\mathrm{GH}$, a gravidade mediana foi de 3 , mas no grupo com artrose, a gravidade mediana foi de 10 . A artrose $\mathrm{AC}$ foi significativamente associada com a idade do paciente ( $p<0,001$, teste de Mann-Whitney). A idade média do paciente no grupo sem artrose AC foi de 33,8 anos, com desvio-padrão de 10,0 anos e mediana de 31,4 anos. No grupo que apresentava artrose $\mathrm{AC}$, a idade foi significativamente maior, com média de 45,7 anos, desvio-padrão de 8,8 anos e mediana de 45,1 anos. A bursite foi significativamente associada com o tempo de trauma $(p=0,022$, teste de MannWhitney), com média de 11,0 anos, desvio-padrão de 9,3 anos e mediana de 7,8 anos.

\section{Discussão}

Em um estudo de biomecânica, Kulig et al. ${ }^{1}$ demonstrou um aumento de $360 \%$ do estresse vertical no ombro em demandas manuais de transferência e propulsão de cadeira de rodas. No entanto, como conclui Alves, ${ }^{10}$ nesses pacientes a dor pode estar relacionada apenas à sobrecarga funcional, e nem sempre há relação com lesões anatômicas.

Encontramos alta prevalência de artrite $A C$ em nossa amostra, com 28 casos (68,3\%), que foi um padrão também observado em outros estudos. ${ }^{5,11}$ Eriks-Hoogland $^{12}$ 


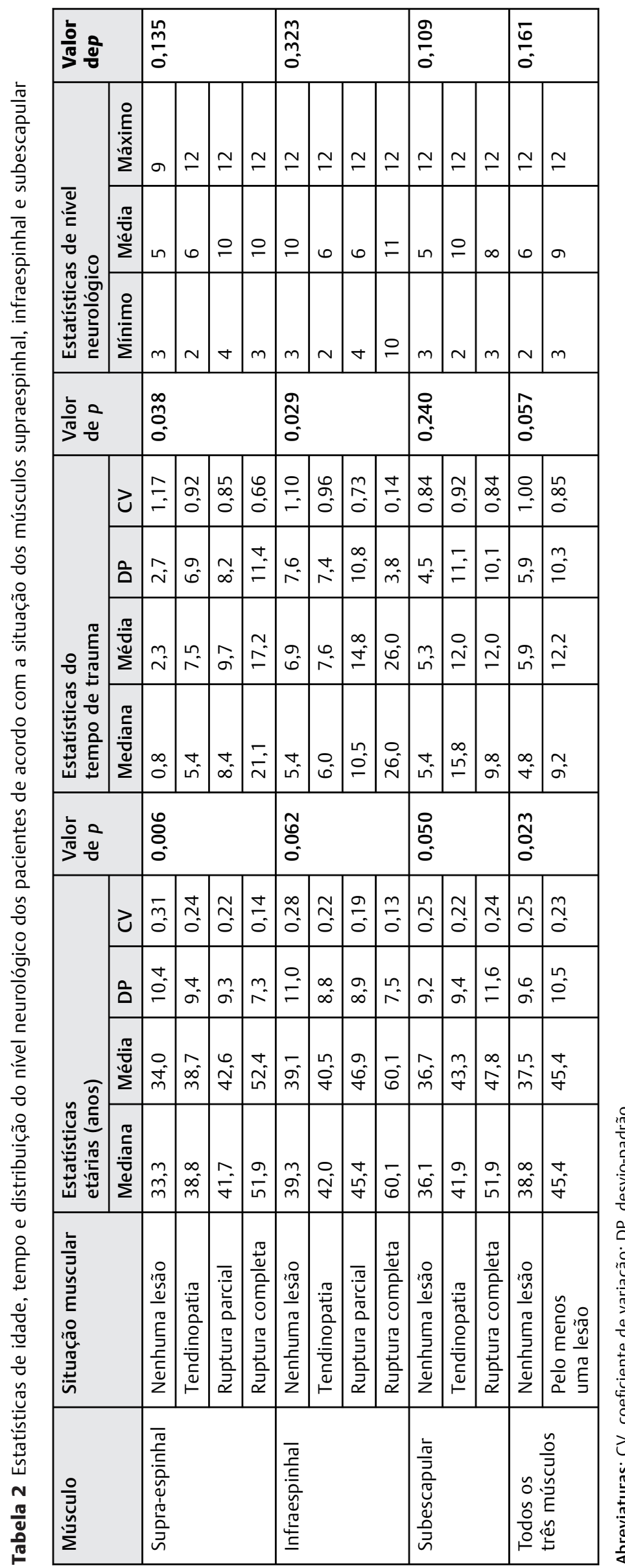


encontrou alta prevalência de artrite AC tanto no grupo de cadeirantes quanto na amostra de controle; no entanto, a gravidade da artrite foi maior em pacientes com LM.

Morrow et al., ${ }^{5}$ em um grupo de 10 cadeirantes manuais, observaram $50 \%$ de presença de lesões supraespinhais e observaram que $60 \%$ dos pacientes tinham lesão em pelo menos um dos tendões do manguito rotador. Em nossa população, os achados foram semelhantes, com $46 \%$ dos pacientes tendo lesões supraespinhais e $56 \%$ em pelo menos um dos tendões.

Para aqueles que apresentaram lesões parciais ou completas, a idade média e o tempo de LM foram 7,9 e 6,3 anos maiores, respectivamente, em comparação com o grupo sem lesões. Ambas as variáveis apresentaram correlação positiva com a gravidade da lesão, com significância estatística (-Fig. 1), conforme encontrado por outros autores. ${ }^{11} \mathrm{O}$ presente estudo, no entanto, não foi capaz de mostrar se um desses fatores foi mais importante para a lesão do manguito rotador, pois ambos apresentaram correlação fraca. Na análise da relação entre o tempo de lesão espinhal e o estado dos tendões da braçadeira, o supraespinhal e o infraspinhal mostraram diferença entre os grupos, enquanto os subescapulares não apresentaram diferença. Esse achado nos permite supor que, ao contrário do supra e do infraespinhal, o trabalho realizado na propulsão da cadeira de rodas não gera sobrecarga no subescapular.

Kulig et al. ${ }^{13}$ realizou um estudo para analisar o efeito do nível de LM na cinética articular do ombro durante a propulsão manual da cadeira de rodas, encontrando uma força vertical superior aumentada nos grupos tetraplégicos, o que poderia levar estruturas subcrômicas a um risco aumentado de compressão. Este parâmetro não foi significativamente diferente entre os grupos de paraplegia alta (T1-T9) e baixa (T10-L3). Nossa amostra continha apenas pacientes com lesão torácica (T2-T12) e, de acordo com o trabalho publicado, não encontramos diferenças estatisticamente significativas entre a gravidade da lesão do manguito rotador nos grupos com alto nível torácico (T1-T6) e baixas (T7-T12). Ambas as observações indicam que as diferenças na capacidade de equilíbrio postural causadas pelo nível de LM não atuam como um fator de sobrecarga significativo na articulação do ombro.

\section{Conclusão}

No presente estudo, observou-se aumento progressivo da gravidade da lesão do ombro à medida que a idade se torna mais avançada e quanto maior o tempo transcorrido desde a ocorrência da LM. No entanto, o nível da lesão torácica aparentemente não interfere com a lesão do manguito rotador.

Conflito de interesses

Os autores não têm conflito de interesses para declarar.

\section{Referências}

1 Kulig K, Rao SS, Mulroy SJ, et al. Shoulder joint kinetics during the push phase of wheelchair propulsion. Clin Orthop Relat Res 1998; (354):132-143

2 Jung HJ, Sim GB, Jeon IH, Kekatpure AL, Sun JH, Chun JM. Reconstruction of rotator cuff tears in wheelchair-bound paraplegic patients. J Shoulder Elbow Surg 2015;24(04):601-605

3 Samuelsson KA, Tropp H, Gerdle B. Shoulder pain and its consequences in paraplegic spinal cord-injured, wheelchair users. Spinal Cord 2004;42(01):41-46

4 Barbetta DC, Lopes AC, Chagas FN, et al. Predictors of musculoskeletal pain in the upper extremities of individuals with spinal cord injury. Spinal Cord 2016;54(02):145-149

5 Morrow MM, Van Straaten MG, Murthy NS, Braman JP, Zanella E, Zhao KD. Detailed shoulder MRI findings in manual wheelchair users with shoulder pain. BioMed Res Int 2014;2014:769649

6 Boninger ML, Dicianno BE, Cooper RA, Towers JD, Koontz AM, Souza AL. Shoulder magnetic resonance imaging abnormalities, wheelchair propulsion, and gender [published correction appears in Arch Phys Med Rehabil 2004;85(1):172. Arch Phys Med Rehabil 2003;84(11):1615-1620

7 American Spinal Injury Association. International standards for neurological classification of spinal cord injury (ISNCSCI). [Accessed 20 May 2019] from: http://asia-spinal-injury.org/wp-content/uploads/2016/02/International_Stds_Diagram_Work-sheet.pdf

8 Bigliani LU, Ticker JB, Flatow EL, Soslowsky LJ, Mow VC. The relationship of acromial architecture to rotator cuff disease. Clin Sports Med 1991;10(04):823-838

9 Goutalier D. Pathologie de la coiffe des rotateurs. In: Encyclopédie Médico Chirurgicale. Paris: Elsevier Masson; 1997350

10 Alves AP, Terrabuio Junior AA, Pimenta CJ, Medina GI, Rimkus CdeM, Cliquet Júnior A. Clinical assessment and magnetic resonance imaging of the shoulder of patients with spinal cord injury. Acta Ortop Bras 2012;20(05):291-296

11 Haffner DL, Hoffer MM, Wiedbusch R. Etiology of children's spinal injuries at Rancho Los Amigos. Spine 1993;18(06): 679-684

12 .06pt?>Eriks-Hoogland I, Engisch R, Brinkhof MW, van Drongelen S. Acromioclavicular joint arthrosis in persons with spinal cord injury and able-bodied persons. Spinal Cord 2013;51(01): 59-63

13 Kulig K, Newsam CJ, Mulroy SJ, et al. The effect of level of spinal cord injury on shoulder joint kinetics during manual wheelchair propulsion. Clin Biomech (Bristol, Avon) 2001;16(09): 744-751 